\title{
Formation , trajectory and distribution variants of the median nerve of the fetus
}

${ }^{1}$ Faculty of Medicine, Titu Maiorescu University, Bucharest

${ }^{2}$ Faculty of Medicine, University "Ovidius" of Constanta

\begin{abstract}
Our study was performed by dissection on a number of 54 nervous trunks of the median nerve of the fetus. We found that the median nerve is always formed from two roots, their joining being at different levels of the upper limb, between the axilla and the elbow. The axilla nerve trunk was formed at the level of the axillary region, in $38.89 \%$ of the cases, in $22.22 \%$ of the cases the union was made at the middle part of the arm, and in $38.89 \%$ of the cases in the elbow. The lateral root of the medial nerve was formed in $55.56 \%$ of cases from a single nerve fascicle, in $44.44 \%$ of cases consisting of two nerve fascicles. The medial root was formed in $61.11 \%$ of cases from a single nerve fascicle, in $38.89 \%$ of the cases being made up of two nerve fascicles. In $27.78 \%$ of cases, the medial root passed behind the axillary artery. Regarding the volume of the two roots, we found that in $44.44 \%$ of the cases, the lateral root was more voluminous, in $27.78 \%$ of cases, the median root was larger and in $27.78 \%$ of cases, the two roots were approximately equal. We have encountered situations where a ramification for the forearms muscles emerged from the lateral root. Occasionally, a ram for the brachial muscle was detached from the medial root, and from the lateral root a ram for the biceps muscle, both muscles receiving branches also from the musculocutaneous nerve. We have encountered a single case where the median nerve inches the anterior muscles of the arm, missing the musculocutaneous nerve. In cases of low joining of the roots, we have encountered cases where a lateral root formed a ram for forearm muscles. The
\end{abstract}

\section{Ioan Sorin Tudorache}

Faculty of Medicine, "Titu Maiorescu" University Bucharest, Romania anastomoses between the two median nerve roots can sometimes be located just above their union or anterior to the lower portion of the axillary artery. In one case, we encountered between the two roots, above their union, the existence of three oblique anastomoses, the two upper ones from the lateral to the medial root, and the third from the medial root to the lateral root. Common are anastomoses between the roots of the roots and the root on the opposite side. The most common are the anastomosis between the medial fascicle of the lateral root and the medial root of the median nerve. In one case, we encountered a double overlap between the musculocutaneous nerve and the lateral nerve root. In one case, we encountered a strong anastomosis between the medial nerve fascicle of the medial root and the radial nerve. Common and at all levels of the upper limb are the anastomoses between the median and ulnar nerves. In the case of a low union of the two median roots, we encountered anastomoses between a root of the root and the ulnar nerve, or between a root and the ulnar nerve. I encountered a single case with an anastomosis, MartinGruber, which was previously passing through the ulnar and interos-like arteries and from which the anterior forearm muscles were detached.

Keywords: median nerve - anatomical variants

\section{Introduction}

Of all the terminal branches of the brachial plexus, the median nerve presents the most anatomical variations in its formation, tract, branch, and anastomoses with the other terminal branches of the plexus. Classically, the median nerve is formed from two roots, medial (Radix medialis nervi mediani ) and lateral (Radix lateralis nervi mediani ), which will unite in the "V", anterior to the axillary artery, 
its fibers coming from the anterior branches of the cervical rachis, nerves $\mathrm{C} 6, \mathrm{C} 7, \mathrm{C} 8$ ) and the first thoracic (T1) $[1,2,3,4,5,6,7,8]$. The lateral root is formed of nerve fibers from the $\mathrm{C} 5, \mathrm{C} 6, \mathrm{C} 7$ nerves, and the fiber mesh root from the $\mathrm{C} 8$ and $\mathrm{T} 1$ nerves [5]. The median nerve passes the lower part of the axillary region, being laterally disposed on the axillary artery, which it crosses before, and descends on the medial face of the arm, crosses the elbow, descends vertically on the median line of the forearm, passes below the flexor retinas reaching the hand, which is divided into its terminal branches $[1,2,3,8]$. The median nerve presents anastomoses between its roots at different levels: in the axilla, when the union of the two roots takes place at this level [1], in the lower arm or the elbow bundle, when the root joining is below $[1,6]$.

The median nerve has the most of its anastomoses with the ulnar nerve: at the level of the forearm $[1,2,9,10,11]$, anastomosis known as Martin-Gruber or palm of the hand where there are two superficial and deep anastomoses [2]. The anastomoses with the musculocutaneous nerve are mentioned by [1,2,12], [2] also describing the possibility of anastomosis between the median and radial nerves.

In the absence of the musculocutaneous nerve, the innervation of the muscles of the arm is provided by the median nerve $[10,11,13]$.

\section{Material and method}

Our study was performed by dissection on formalin preserved fetal human corpses, aged 4-8 months. Some corpses were injected with red plastic mass in the artery (Technovit 7143), highlighting the median nerve trajectory and ratios with neighboring arteries. There were 54 fetal nerve trunks followed by the anatomical characteristics of the nerve roots, the path of the median nerves within the axilla, the arm, the elbow and the forearm, and the existing anastomoses between the median nerve and the neighboring nerves.

\section{Results}

We have found that the median nerve is always formed by two roots, originating in the medial and lateral fascicles, originating in the lateral nerve fascicle of the brachial plexus. The union of the two roots occurs at different levels of the upper limb, between the axilla and the elbow fold. The axillary nerve trunk was formed at the level of the axillary region, in 21 cases $(38.89 \%$ of cases), in 12 cases (57.14\% of cases) on the medial side of the axillary artery, in 6 cases $(28.57 \%$ of cases) on the anterior face and in 3 cases (14.29\% of cases) on the lateral side of the axillary artery. In 12 cases $(22.22 \%$ of cases) the union of the two roots was made at the level of the brachial region, in 9 cases ( $75 \%$ of cases) on the medial side of the brachial artery and in 3 cases $(25 \%$ of cases) on the lateral side of the brachial artery. In the other 21 cases $(38.89 \%$ of the cases) the union of the roots was made within the fold of the elbow, in 12 cases $(57.14 \%$ of cases) on the medial face of the brachial artery, in 6 cases $(28.57 \%$ of cases) on the anterior side of the brachial artery, and in 3 cases (14.29\% of cases) on the lateral side of the brachial artery.

The medial nerve root was formed in 30 cases (55.56\% of cases) from a single nerve fascicle, in 24 cases (44.44\% of cases) consisting of two nerve fascicles, $(50 \%$ of cases) on the anterior face of the axillary artery, in 10 cases ( $41.67 \%$ of cases) on the lateral side of the axillary artery and in 2 cases $(8.33 \%$ of cases ) on the medial face of the axillary artery. 


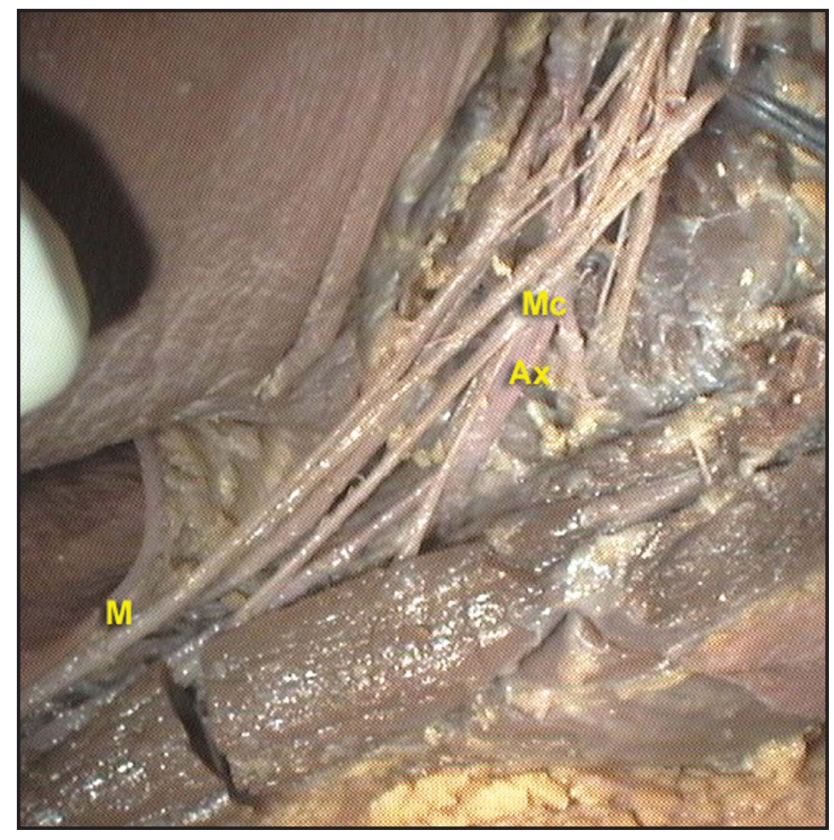

Figure 1. The two median roots are joined to the middle of the arm, the medial of to the brachial artery; The medial root is composed of two medial and lateral bundles medial joining by the axillary artery; The lateral root, also formed of two bundles joining laterally to the axillary artery, crosses the axillary artery in advance to join with the medial root; From the midline of the lateral root an anastomosis come undone for the medial root; $M$ n. median; Mc: $n$. Musculocutan; Ax: a. Axillary.

Regarding the volume of the two roots of the mediaRegarding the volume of the two roots of the median nerve, we found that in 24 cases $(44.44 \%$ of cases), the lateral root was more voluminous, in 15 cases $(27.78 \%$ of cases) the medial root was more voluminous and in 15 cases $(27.78 \%$ of cases) the two roots were approximately equal. In cases where the two roots of the medial nerve join at the axillary level or even at the brachial level, it usually retains its anatomical tract, justifying its name. In the case of uniting the roots in the fold of the elbow, they may have a particular path, especially the lateral root. Thus, in such a case, above the fold of the elbow, the lateral root passes medially to the brachial artery and posterior to the medial root, postero-medial to it, until their union to form the median trunk. Another case of nervous trunk formation in the fold of the elbow, in which the medial root is made up of two fascicles which are joined medial to the axillary artery, the lateral fascicle being initially located laterally to the axillary artery, crosses it over and joins with the medial nerve fascicle on the medial part the brachial artery, above the crossing, the two nerve fascicles having the axillary artery there between; the lateral root was initially located laterally to the axillary artery, crosses it anteriorly and passes it medially.

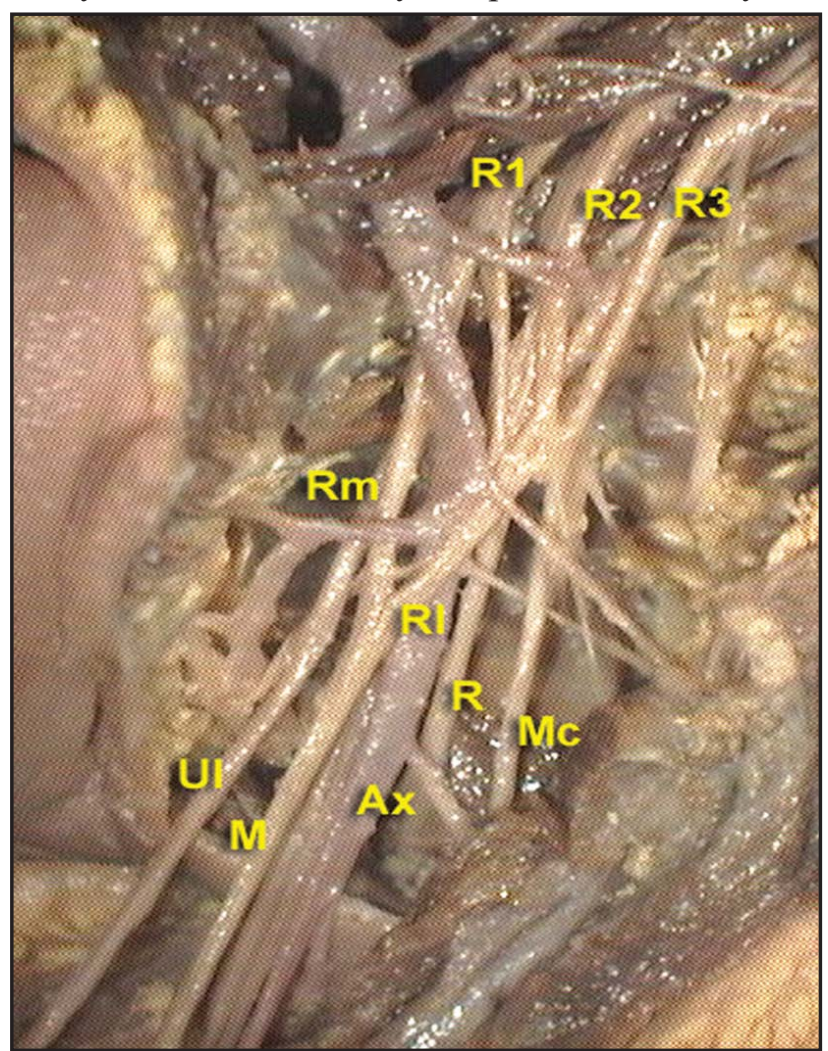

Figure 2. Medial nerve consisting of two roots: the medial, consisting of a single nerve fascicle, passes behind the axillary artery, and the lateral root is formed by two medial nerve fascicles (R2) and later (R3) beads that join laterally to the axillary artery; The lateral root formed previous passes through the axillary artery, medialally joining to the axillary artery with the medial root, forming the trunk of the median nerve, which continues its downward, medial path to the axillary and brachial arteries; A strong anastomosis for the radial nerve (R1) is detached from the medial, super-medial fascial artery; Rm: the media root; $R 1$ : lateral root; $R: n$ radial; Axis: a. Axillary; Ui: $n$. Ulnar; M: n. Median. 


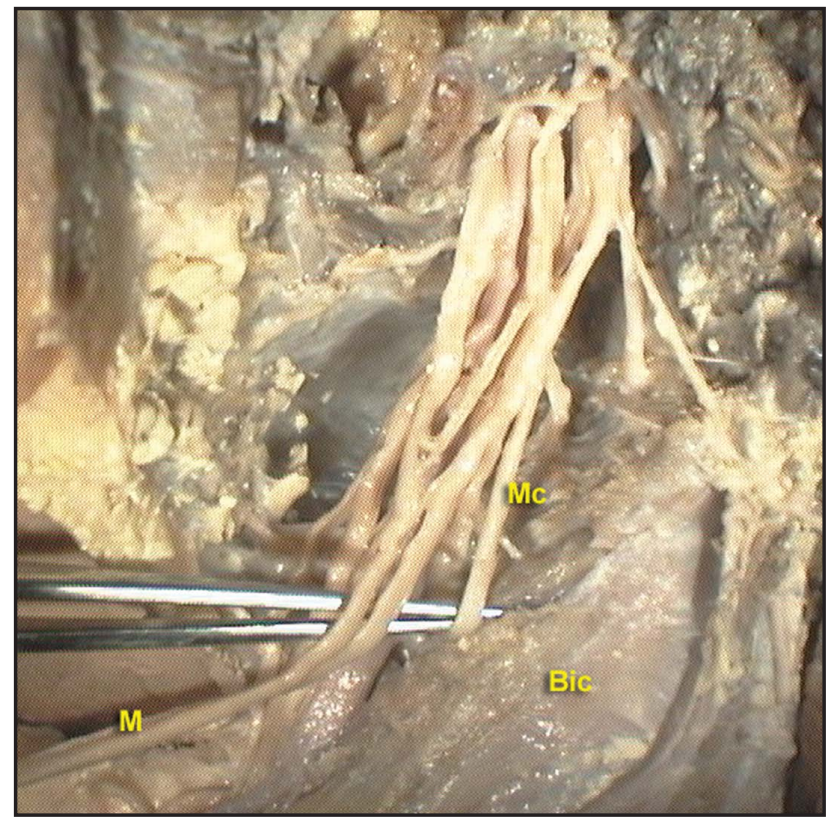

Figure 3. The medial root is made up of two nerve fascicles, which are medial to the axillary artery, continuing this trajectory to the fold of the elbow level; The lateral root is also formed of two fascicles which unit previous to the axillary artery; above the fold of the elbow, the lateral root passes medially to the brachial artery and posterior to the medial root, postero-medial; Strong anastomosis between the medial nerve fascicle of the lateral root and the medial root in the upper part of the arm; $M$ : the two root of the $n$.medial in the upper fold of the elbow; Mc: n. Musculocutan; Bic: me. Biceps brachial

Another particular case is that in which the lateral root is formed by two fascicles (medial and lateral) disposed laterally to the axillary artery, joining anterolateral to the axillary artery, maintaining a demarcation area between them, up to the level of the union with the medial root, practically joining each other. We have encountered situations where a branch for the forearm muscles emerged from the lateral root. Sometimes a branch for the brachial muscle was detached from the medial root, and from the lateral root a branch for the biceps muscle, both muscles receiving branches from the musculocutaneous nerve. I have encountered a single case where the median nerve supplies the anterior muscles of the arm, missing the musculocutaneous nerve.

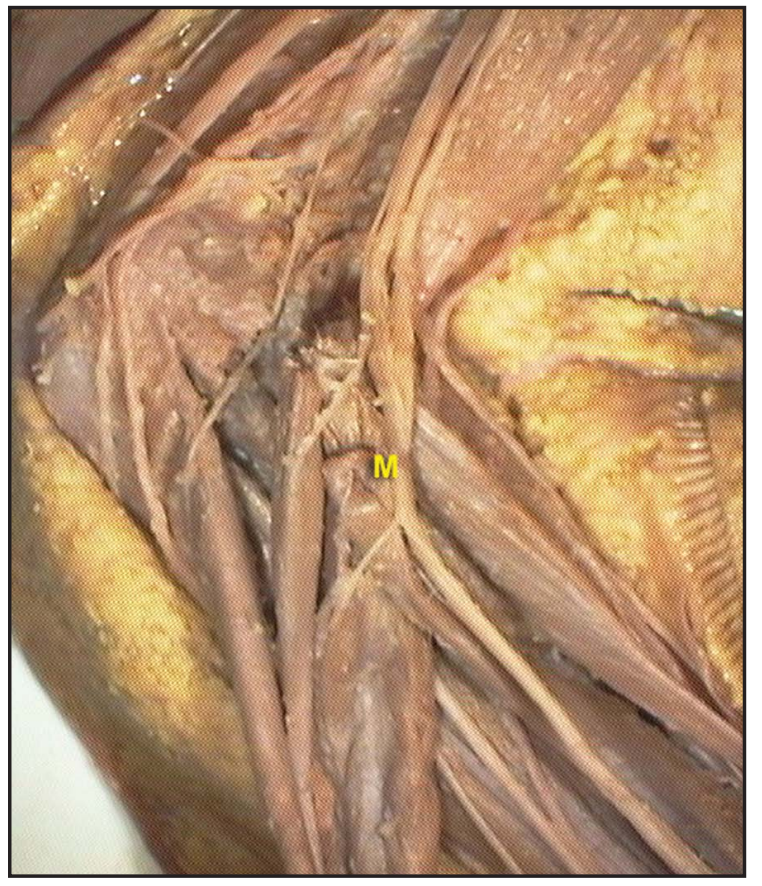

Figure 4.From the lateral root of the median nerve a ram for forearm muscles is detached, after joining the two medial nerve roots, a ram for forearm muscles is also detached.

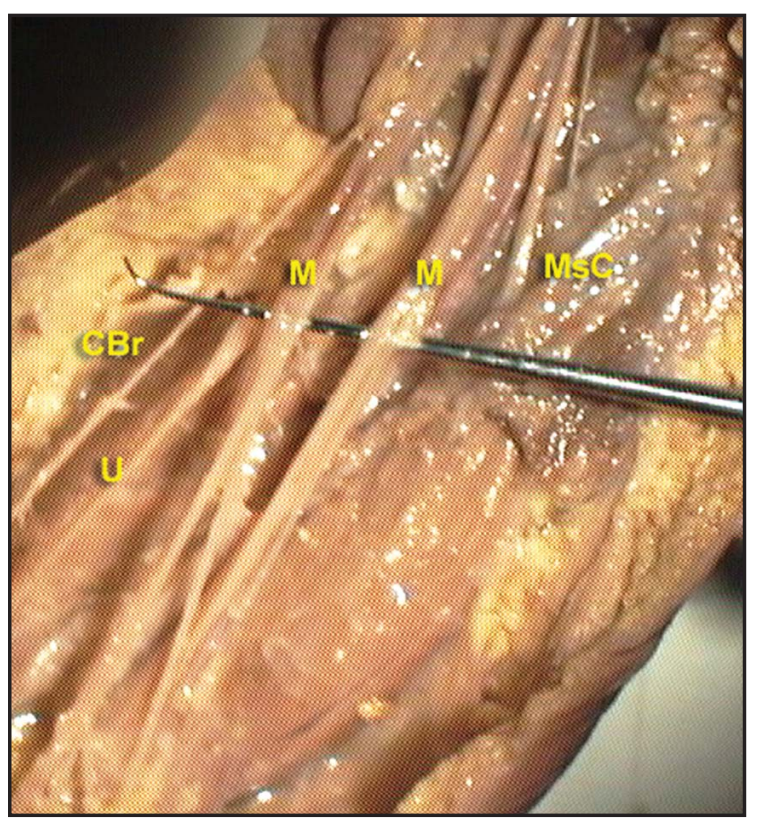

Figure 5 From the medial root of the median nerve, a ram for the brachial muscle is detached, and from the lateral root a ram for the biceps muscle, both muscles receiving branches from the musculocutaneous nerve; $M, M$ : the roots of the median nerve; MsC: $n$. Musculocutaneous; $C B r$ : $n$ antebrahial cutaneous; $U: n$. Ulnar. 
In cases of lower joining of the roots, we have encountered cases where a lateral root formed a branch for the forearm muscles.

The median nerve presents numerous anastomoses, at all levels of the upper limb. The anastomoses between the two median nerve roots can sometimes be located just above their union or anterior to the lower portion of the axillary artery.

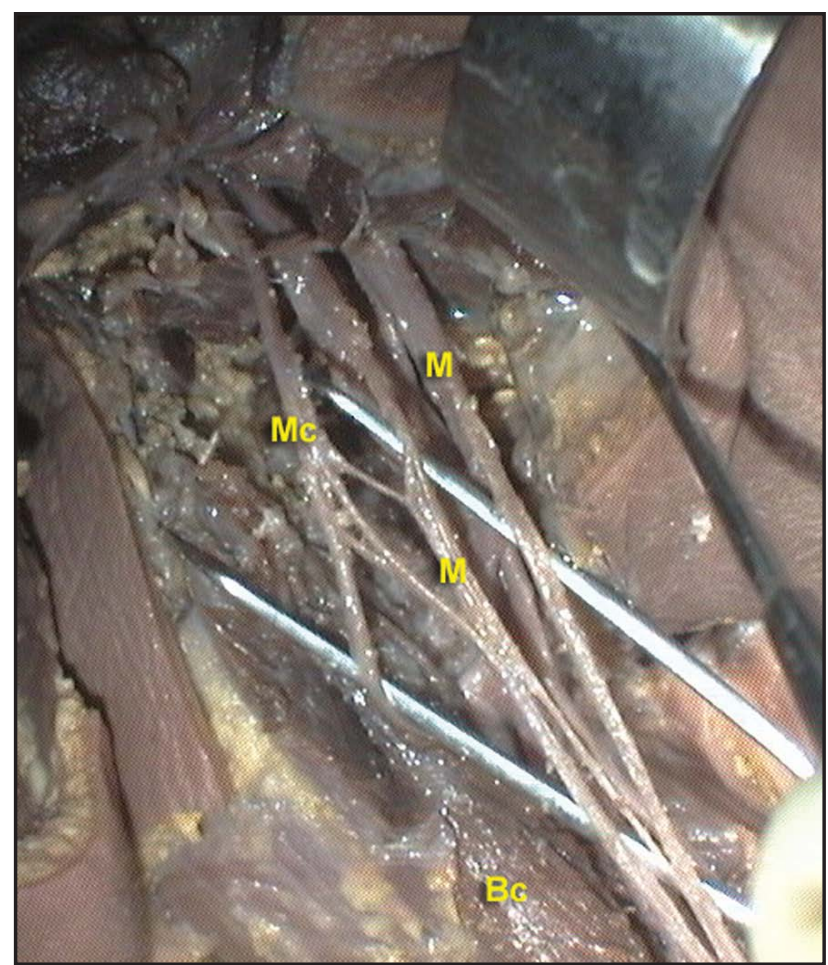

Figure 6. The two roots of the median nerve join together in the fold of the elbow; Double anastomosis superimposed between the musculocutaneous nerve and the lateral root of the median nerve, the lower anastomosis being double at origin; Oblique anastomosis between the lateral and medial nerve roots; $M, M: n$. Median roots; Mc: $n$. Musculocutan; Bc: mrs. Biceps brahial.

In one case I encountered, between the two roots and above their union, the existence of three oblique anastomoses, the two upper ones from the lateral to the medial root, and the third from the medial root to the lateral root.

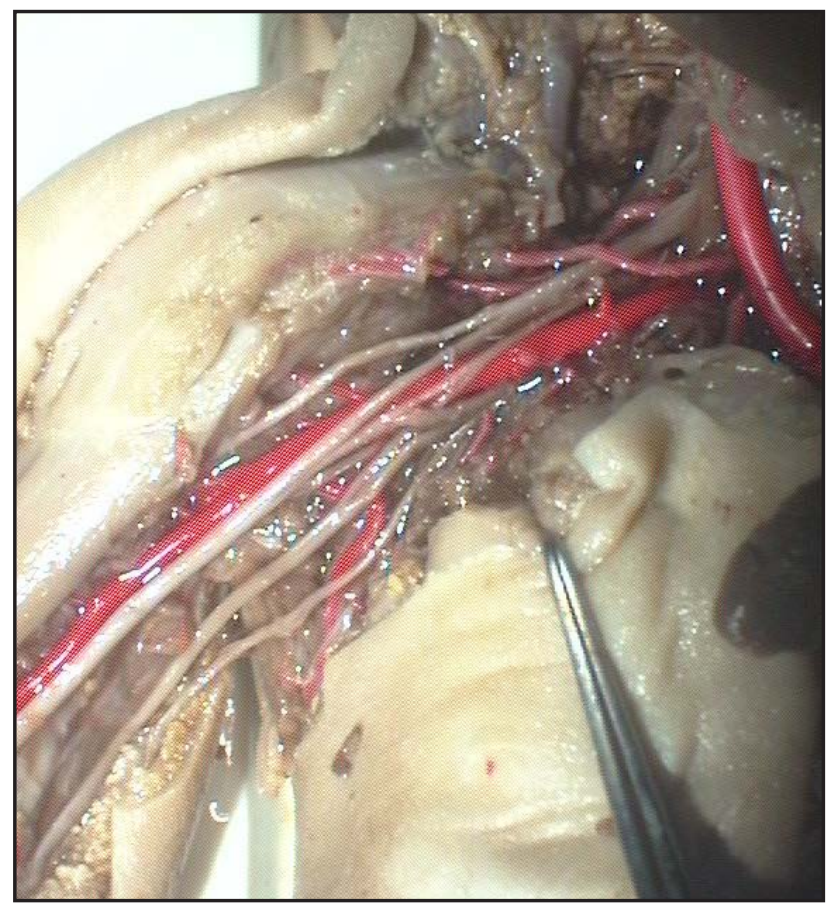

Fig. 7. Median nerve formed from two roots that are medial to the axillary artery, the lateral root being slightly more voluminous; Strong anastomosis between the medial root and the ulnar nerve, anastomosis passing behind the artery.

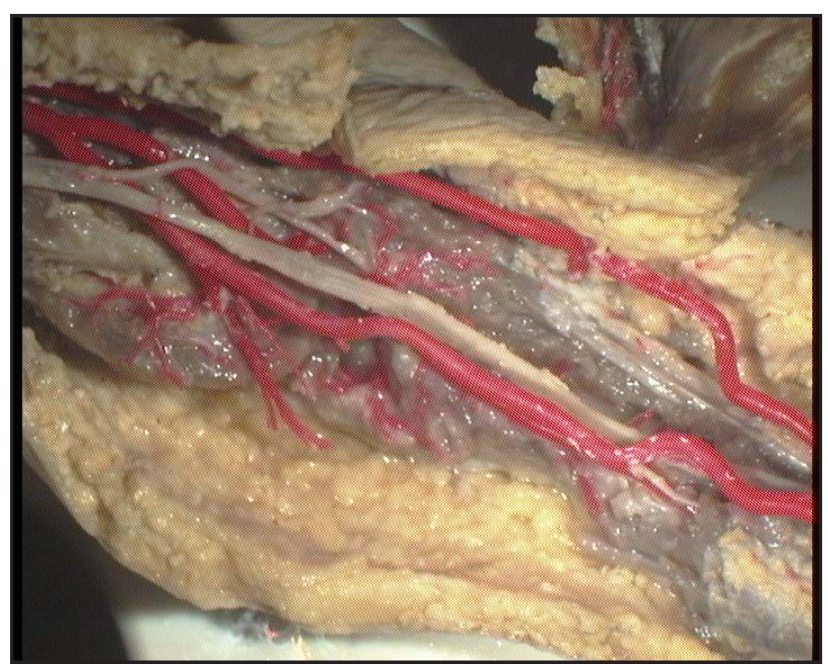

Fig. 8. Anastomosis Martin-Gruber, which passes anterior to the ulnar and interosseous arteries and from which the anterior antebrachial muscles are detached 
Commonly are anastomoses between the roots of the roots and the root on the opposite side. I have encountered anastomosis between the lateral cord of the brachial plexus and the medial root of the median nerve, which can be considered the second nerve fascicle of the medial root with the axillary artery between them. The most common are the anastomosis between the medial nerve fascicle of the lateral root and the medial root of the median nerve. In one case, we encountered a double anastomosis, superimposed between the musculocutaneous nerve and the medial nerve root, the lower anastomosis being double at the origin, this case also presenting an oblique anastomosis between the medial and nerve root of the medial nerve, the two roots united to the fold of the elbow. In one case, we encountered a strong anastomosis between the medial fascicle of the medial root and the radial nerve. Common and at all levels of the upper limb are the anastomoses between the median and ulnar nerves. In case of low union of the two roots of the median, we encountered anastomoses between a root of the root and the ulnar nerve, or between a root and the ulnar nerve, anastomoses passing anterior or posterior to the axillary or brachial artery. We encountered a single case with a Martin-Gruber anastomosis, which was previously passing through the ulnar and interosseous arteries and from which anterior antebrahial musclethreads were emerging.

\section{Discussions}

When the joining of the two roots of the medial nervWhen the joining of the two roots of the medial nerve occurs before the axillary artery, the roots are located on both sides of the artery that is flanked by them.

When the roots union occurs medially or laterally (less frequently) of the axillary artery, one of the two roots crosses anteriorly the axillary artery, more commonly the lateral root, to medially join the medial root. In 12 cases ( $22.22 \%$ of cases), the medial nerve formation occurred on the anterior face of the axillary or brachial artery (in the case of low medial nerve formation), in 9 cases ( $16.67 \%$ of cases) on the lateral side of these arteries and, most frequently, in 33 cases $(61.11 \%$ of cases), on their medial side.

According to $[1,2,4]$, in adult, the union of the roots is made anterior to the axillary artery, [1] quoting the possibility of their union at the level at the arm and forearm, without specifying the frequency of these variants of the formation of the median nerve. [3] describes the formation of the median nerve within the lower $1 / 3$ of the axillary artery, anterior or lateral to the artery. [10] finds that adult medial nerve formation occurs in the axilla in $68.33 \%$ of cases, percentage higher than our percentage by $29.94 \%$. In these cases he assess that the union of the roots was performed, in $76.67 \%$ of cases, lateral to the artery (71.11\% higher than our percentage), the anterior face formed in $20 \%$ of the cases (percentage higher than ours with $8.89 \%$ ), and in $3.33 \%$ of the cases, the union of the roots was done medial to the artery (a percentage lower than ours by $53.81 \%$.) Also, [10] finds that the median nerve was formed at the level of the brachial region in 20 of these cases, the medial nerve formation takes place on the lateral side of the artery in $96.67 \%$ of the cases, a percentage greater than the one found by us by $71.67 \%$ and in $3.33 \%$ of cases being formed on the medial side of the artery, a percentage lower than ours with $71.67 \%$.

The lateral root was found to be more bulky in $16.66 \%$ of cases in relation to medial root, but the two roots are formed in similar percentages of one or two nerve bundles, the lateral one being formed by two bundles with just 0.87 percent more than the medial root. For [1], the lateral root is more bulky, like us $[1,2,3,4,5,6,12]$ assess that the median nerve is formed by two roots. $[14,15]$ find that the median nerve consists of three branches. [10] finds that the median nerve consists of two roots in $88.33 \%$ of cases, in $11.66 \%$ of cases consisting of three roots. We do not rule out the possibility of forming the median nerve from three roots, given that some anastomoses of the roots originate at the level of a brachial plexus nerve bundle and end near the union of the medial and lateral of the medial nerve roots, thus can be considered as a third root, but can also be considered as a nerve fascicle of one of the roots or as 
an anastomosis.

The possibility of anastomosis between the medial root nerve of the medial nerve and the radial nerve was not found in the literature I was able to consult.

Anastomoses of the median nerve with the musculocutaneous nerve are quoted by $[1,2,12],[10]$ found in $11.67 \%$ of the cases.

The possibility of innervation of the anterior brachial muscles by the medial nerve is mentioned by $[10,11,12,13],[10]$ also mentioning the possibility of absence of the musculocutaneous nerve, its motor innervation being taken up by the median nerve. Martin-Gruber anastomosis was encountered in only one case (1.85\% of cases), [9] found it in $23.6 \%$ of cases, Mannerfeld, quoted by [9], in 15\% of cases, and Crutchfield and Gutmann, quoted by [9], on an electrophysiological study, found it in $28 \%$ of the general population and $62 \%$ of the cases in a family where one of them had this anastomosis.

\section{Conclusions}

The median nerve presents the most variants of all the terminal branches of the brachial plexus, especially the variants of formation and anastomosis between its roots and the neighboring nerves. We have not found in literature a study of the median nerve on the fetuses and, for this reason, we have compared the anatomical characteristics of the nerve described in the adults.

The differences encountered were due to the subsequent development of vascular and nerve elements and the increase in volume of the muscles with which the median nerve is in relation, as well as to the increase in the size of the upper limb segments with age.

\section{References}

1. Testut, L. (1922). Nerf médian. In: Traité d'anatomie humaine. Tome troisième. Sistème nerveux périphérique. Organes des sens. Appareil de la respiration et de la phonation. Ed. Gaston Doin, Paris, (pp. 172-178)

2. Rouvière, H. \& Delmas, A. (1997). Nerf médian. In: Anatomie humaine. Descriptive, topographique et fonctionelle. Tome 3. Membres. Sistème nerveux central. $14 \mathrm{e}$ édition. $\mathrm{Ed}$. Masson, Paris, (pp. 187-193)

3. Standring, S. (2005) Gray's Anatomy. The Anatomical Basis of Clinical Practice. Ed. Elsevier Churchill Livingstone, Edinburg. (pp. 848; 864; 885-886;932)

4. Bonnel, F. (1991). Nerf médian. In: JP Chevrel Anatomie clinique. 1. Les membres, Ed SpringerVerlag, Paris. (pp 9-33;75-81; 133; 199-201; 263-265)

5. Nagwani, M. (2014). A Variant Course of Lateral Root of Median Nerve: Embryological Basis. Asian Journal of Medical Sciences (E-ISSN 2091-0576; P-ISSN 2467-9100). 5(2), 146-150.

6. Singh, B., Gupta, G.S. \& Gorakhpur, M.A. (2013). Atypical Intercommunication in the Roots of Median Nerve- a Rare Variation. Research Paper Medical Science, 2(12), 182-183

7. Moore, K. \& Dalley, A. (2001) Anatomie Médicale. Aspects fondamentaux et applications cliniques. Ed. De Boeck Université, Bruxelles, (pp. 708-717; 730-731; 757-759; 774-776)

8. Bordei, P. \& Ulmeanu, D. (2002). Nervul median. In: Anatomia descriptivă a membrelor. Ed. Ex Ponto, Constanţa, (pp. 174-179)

9. Shu, H., Chantelot, C., Oberlin, C., ALNOT, J.-Y. \& Shao, H. (1999). Etude anatomique et revue de la litterature a propos de l'anastomose de Martin Gruber. Morphologie. 83(260), 71-74.

10. Pattanshetti, S.V., Jevoor, P., Shirol, V., Dixit, D. \& Bhimalli, S. (2012). A study of the formation and branching pattern of brachial plexus and its variations in adult human cadavers of north 
Karnataka. Journal of the Scientific Society. 39(2), 70.

11. Gumusburun, E. \& Adiguzel, E. (2000). A variation of the brachial plexus characterized by the absence of the musculocutaneous nerve: a case report. Surg Radiol Anat. 22(1), 63-65.

12. Munjamkar, P., Kamdi, N. \& Meshram, M. (2015). Abnormal formation of Median nerve in Arm with lateral cord piercing the Coracobrachialis. Research Chronicle in health sciences. 1(1), 3538.

13. Nakatani, T., Tanaka, S. \& Mizukami, S. (1997). Absence of the musculocutaneous nerve with innervation of coracobrachialis, biceps brachii, brachialis and the lateral border of the forearm by branches from the lateral cord of the brachial plexus. J Anat. 191 ( Pt 3), 459-460.

14. Sargon, M., Uslu, S., Celik, H. \& Akşit, D. (1995). A variation of the median nerve at the level of brachial plexus. Bulletin de l'Association des Anatomistes. 79(246), 25-26.

15. Goyal, N., Harjeet \& Gupta, M. (2005). Bilateral variant contributions in the formation of median nerve. Surg Radiol Anat. 27(6), 562-565. doi: 10.1007/s00276-005-0023-6.

16. $* * * * * * * *(1998)$ N. medianus. In: Terminologia Anatomica. International Anatomical terminology. FCAT. Federative Committee on Anatomic Terminology. Ed.Thieme Stuttgart. New York, 138 